\title{
THE NORMALITY OF CERTAIN SUBGROUPS OF ELEMENTARY SUBGROUPS OF STEINBERG GROUPS OVER RINGS
}

\author{
JAMES F. HURLEY
}

\begin{abstract}
This paper amends the approach used in an earlier paper to construct, from ideals in the Chevalley algebra $L_{R}$ over a commutative ring $R$ with identity, normal subgroups of the elementary subgroup $G_{R}^{1}$ of Steinberg's twisted group corresponding to $L$, a finite dimensional simple Lie algebra over the complex field. The set of normal subgroups so constructed turns out to be in one-to-one correspondence with the set of equivalence classes of ideals of $R$ under an equivalence relation defined in terms of the underlying automorphism of $R$ of order 2 .
\end{abstract}

1. Introduction. In [3], to which the reader is referred for all notation not specified here, the same procedure developed in [2] was used with the aim of creating normal subgroups of $G_{R}^{1}$ corresponding to ideals $I$ in the Chevalley algebra $L_{R}$ of the Lie algebra $L$ over $R$. R. W. Carter has pointed out to the author that the procedure of [2] fails in general to define a normal subgroup in the context of [3], since the elements $x_{r}(t)$ which lie in $U_{R}^{1}$ or $V_{R}^{3}$ do not generate the group $G_{R}^{1}$. In the present paper, a modification of the approach found in $[3, \S 4]$ is used to construct a normal subgroup $G_{I}^{1}$ of $G_{R}^{1}$ corresponding to an ideal $I$ of $L_{R}$. The author would like to acknowledge and thank Professor Carter for the suggestion that a condition of the form $u e_{s}+\bar{u} e_{\bar{s}} \in I$ would be needed in the definition of $G_{I}^{1}$ (see (5) and (6) below).

While such a normal subgroup could be described as the subgroup of $G_{R}^{1}$ generated by a certain set of elements in the manner of [2], these elements are much more complicated than the corresponding generators of $G_{I}$ in [2] so that such a description becomes quite unwieldy. The normal subgroup $G_{I}^{1}$ in [2] however is the normal closure of the subgroup generated by all elements $x_{r}(t)$ where $t e_{r} \in I$. It is this fact which serves as the point of departure for the approach taken in the present paper. We define $G_{I}$ to be the normal closure of the elements of type (4), (5), and

Received by the editors March 30, 1973.

AMS (MOS) subject classifications (1970). Primary 20G35, 17B20, 20H25; Secondary 20D15, 20F40.

Key words and phrases. Chevalley group, Steinberg group, elementary subgroup, root systems, Lie algebras.

(C) American Mathematical Society 1974 
(6) below. The problem, then, is to determine to what extent distinct ideals of $L_{R}$ gives rise to distinct normal subgroups of $G_{R}^{1}$. If $I \cap E_{R}=J E_{R}$ and $I^{\prime} \cap E_{R}=J^{\prime} E_{R}$, then we show that $G_{I}^{1}=G_{I^{\prime}}^{1}$ if and only if $J \cap \bar{J}=J^{\prime} \cap \bar{J}^{\prime}$. Here $E_{R}=R \otimes_{Z} E_{Z}$ where $E_{Z}$ is the free abelian group on the Chevalley basis elements not in $H$. We thus obtain a bijective correspondence between the set of normal subgroups $G_{I}^{1}$ and the set of equivalence classes of ideals of $R$ modulo the equivalence relation on the set of ideals of $R$ defined by the condition $J^{\prime} \cap \bar{J}^{\prime}=J \cap \bar{J}$.

2. Generators for $G_{R}^{1}$. We assume that the Lie algebra $L$ has a symmetry $r \rightarrow \bar{r}$ of order 2 of its Coxeter-Dynkin diagram, so that $L_{R}$ has a semiautomorphism of order 2 [6, Lemma 3.2]. We also assume that $R$ has an automorphism $t \rightarrow \bar{t}$ of order 2. Then $\sigma: G_{R} \rightarrow G_{R}$ given by $\sigma\left(x_{r}(t)\right)=x_{\bar{r}}(\bar{t})$ is an automorphism. $G_{R}^{1}$ is the subgroup of $G_{R}$ generated by $U_{R}^{1}$ and $V_{R}^{1}$, which are the respective intersections of the fixed point group of $\sigma$ with the maximal unipotent subgroups $U_{R}$ and $V_{R}$ generated by the elements $x_{r}(t)$ where $r$ runs respectively over the positive and negative roots. Then $G_{R}^{1}$ is generated [6, Lemma 4.6] by elements of the forms:

(1) $x_{r}(t)$, where $r=\bar{r}, t=\bar{t}, r \neq s+\bar{s}$ for any root $s$;

(2) $x_{r}(t) x_{\bar{r}}(\bar{t})$, where $r<\bar{r}$, and $r+\bar{r}$ is not a root;

(3) $x_{r}(t) x_{\bar{r}}(\bar{t}) x_{r+\bar{r}}(v)$, where $r<\bar{r}<r+\bar{r}$, and $v+v=N_{r, \bar{r}} t \bar{t} \quad\left(\left[e_{r}, e_{\bar{r}}\right]=\right.$ $N_{r, \bar{r}} e_{r+\bar{r}}$. These generators arise only in case $L$ is of type $A_{n}, n$ even. In this case, we assume that 2 is a unit in $R$. Note that in this case, no generators of type (1) appear [6, p. 877].

3. Normal subgroups of $G_{R}^{1}$. Let $I \nsubseteq H_{R}$ be an ideal of $L_{R}$. Let $G_{I}^{1}$ be the normal closure in $G_{R}^{1}$ of all elements of the forms:

(4) $x_{s}(u)$, where $u e_{s} \in I, u=\bar{u}$, and $s=\bar{s}$;

(5) $x_{s}(u) x_{\bar{s}}(\bar{u})$, where $u e_{s}+\bar{u} e_{s} \in I, s<\bar{s}$, and $s+\bar{s}$ is not a root;

(6) $x_{s}(u) x_{\bar{s}}(\bar{u}) x_{s+\bar{s}}(w)$, where $u e_{s}+\bar{u} e_{\bar{s}} \in I, w+w=N_{s, \bar{s}} u \bar{u}$, and $s<\bar{s}<s+\bar{s}$.

Then, of course, $G_{I}^{1}$ is a normal subgroup of $G_{R}^{1}$ which corresponds to the ideal $I$ of $L_{R}$. The natural question that arises now is to what extent distinct ideals of $L_{R}$ give rise to distinct normal subgroups of $G_{R}^{1}$. The first observation to be made is $[1,3.4]$ that if $I$ is an ideal of $L_{R}$, then $I \cap E_{R}=E_{J}=J E_{R}$ for some ideal $J$ of $R$. $J$ is of course uniquely determined by $I$, but not conversely: $I \cap H_{R}$ may coincide with or properly contain $H_{J}=J H_{R}$. Thus we see that if $I$ and $I^{\prime}$ are two ideals of $L_{R}$ which determine the same ideal $J$ of $R$, then $G_{I}^{1}=G_{I^{\prime}}^{1}$, but this sufficient condition is not necessary. A necessary and sufficient condition is given by the following theorem.

THEOREM 1. $G_{I}^{1}=G_{I^{\prime}}^{1}$ if and only if $J \cap \bar{J}=J^{\prime} \cap \bar{J}^{\prime}$ where $I \cap E_{R}=J E_{R}$ and $I^{\prime} \cap E_{R}=J^{\prime} E_{R}$. 
Proof. Suppose first that $J \cap \bar{J}=J^{\prime} \cap \bar{J}^{\prime}$. Consider type (4) generators $x_{s}(u)$ for $G_{I}^{1}$ where $u=\bar{u} \in J$. Then $\bar{u} \in \bar{J}$, so $u \in J \cap \bar{J}$, hence $u \in J^{\prime} \cap \bar{J}^{\prime}$. Thus all such generators also belong to $G_{I}^{1}$. For type (5) generators $x_{s}(u) x_{\bar{s}}(\bar{u})$ we have $u e_{s}+\bar{u} e_{\bar{s}} \in J E_{R}$, so $u e_{s} \in J E_{R}$ and $\bar{u} e_{\bar{s}} \in J E_{R}$ from $[3,3.4]$. Then $u \in J, \bar{u} \in J$. Since $\bar{u} \in \bar{J}$, we have $\bar{u} \in J \cap \bar{J}$, and since $\bar{u} \in J$, we have $u=\overline{\bar{u}} \in \bar{J}$, hence $u \in J \cap \bar{J}$, also. Thus both $u$ and $\bar{u}$ are in $J^{\prime} \cap \bar{J}^{\prime}$. Thus all these generators belong to $G_{I^{\prime}}^{1}$. Finally, for type (6) generators $x_{s}(u) x_{s}(\bar{u}) x_{s+\bar{s}}(w)$ we again have $u e_{s}+\bar{u} e_{\bar{s}} \in J E_{R}$, so $u$ and $\bar{u}$ belong to $J \cap \bar{J}=J^{\prime} \cap \bar{J}^{\prime}$. Also then $u \bar{u} \in J \cap \bar{J}=J^{\prime} \cap \bar{J}^{\prime}$, hence $w \in J^{\prime} \cap \bar{J}^{\prime}$. So all these generators are in $G_{I^{\prime}}^{1}$. Hence $G_{I}^{1} \cong G_{I^{1}}^{1}$. But interchanging $G_{I}^{1}$ and $G_{I^{\prime}}^{1}$ and performing the same reasoning, we get $G_{I^{\prime}}^{1} \cong G_{I}^{1}$.

Conversely now, suppose $J \cap \bar{J} \neq J^{\prime} \cap \bar{J}^{\prime}$. Then say $y \in J \cap \bar{J}, y \notin J^{\prime} \cap \bar{J}^{\prime}$. If $y=\bar{y}$ and there are self-conjugate roots $s$, then we have $x_{s}(y) \in G_{I}^{1}$. But $x_{s}(y) \notin G_{I^{\prime}}^{1}$. For the only way to obtain elements of $G_{I^{\prime}}^{1}$ is as products of conjugates of elements of type (4), (5), and (6), or their inverses [5, p. 53]. Now if $u$ and $u^{\prime}$ are in $J^{\prime} \cap \bar{J}^{\prime}$, then $x_{r}(u) x_{r}\left(u^{\prime}\right)=x_{r}\left(u+u^{\prime}\right)$, where $u+u^{\prime} \in J^{\prime} \cap \bar{J}^{\prime}$. So $u+u^{\prime} \neq y$. Also in conjugating any elements of $G_{I}^{1}$, we can conjugate each factor and either lengthen the product, or use the commutator lemma in the form

$$
x_{r}(t) x_{s}(u) x_{r}(-t)=x_{r+s}( \pm t u) x_{s}(-u)
$$

$[7$, p. 24], or use a relation of the form

$$
h_{r}(t) x_{s}(u) h_{r}(t)^{-1}=x_{s}\left(t^{c(s, r)} u\right), \quad \text { or } \quad \omega_{r}(1) x_{s}(u) \omega_{r}(1)^{-1}=x_{w r s}( \pm u),
$$

where $h_{r}(t)=\omega_{r}(t) \omega_{r}(-1), \omega_{r}(t)=x_{r}(t) x_{-r}\left(-t^{-1}\right) x_{r}(t), t$ a unit in $R$ (cf. $\left[6,5.1\right.$ and 7.3]). In the second to last case, observe that if $u \in J^{\prime} \cap J^{\prime}$ then $t^{c(s . r)} u \in J^{\prime} \cap \bar{J}^{\prime}$ also, so $y \neq t^{c(s . r)} u$. In the case involving the commutator lemma, observe that if $u \in J^{\prime} \cap \bar{J}^{\prime}$, then $\pm t u \in J^{\prime} \cap \bar{J}^{\prime}$, which is an ideal since $\bar{J}^{\prime}$ is the image of the ideal $J^{\prime}$ of $R$ under a ring automorphism. Thus no $\pm t u$ will be $y$. Thus there is no way to obtain $x_{s}(y) \in G_{I^{\prime}}^{1}$. If there are no self-conjugate roots, then we can apply the reasoning just used to $x_{s}(y) x_{\bar{s}}(\bar{y})$ or $x_{s}(y) x_{\bar{s}}(\bar{y}) x_{s+\bar{s}}(w)$ in $G_{I}^{1}$, and reach the conclusion that the respective one of these elements is not in $G_{I^{\prime}}^{1}$, since $y e_{s}+\bar{y} e_{\bar{s}} \notin I^{\prime}$. Thus $G_{I^{\prime}}^{1} \neq G_{I}^{1}$. This completes the proof.

Now let us define an equivalence relation on the set $\mathscr{I}(R)$ of ideals of $R$ by $J \sim J^{\prime}$ if and only if $J \cap \bar{J}=J^{\prime} \cap \bar{J}^{\prime}$. It is clear that this is an equivalence relation, and Theorem 1 yields the following corollary.

COROLlARY. The set of normal subgroups $G_{I}^{1}$ defined above is in one-toone correspondence with the set $\mathscr{I}(R) / \sim$ of equivalence classes of ideals of $R$.

What additional information do our results give about the normal structure of $G_{R}^{\prime}$ ? As one example, we obtain the following result along the lines of Satz 2 of [4]. 
THEOREM 2. Suppose $G_{R}^{1}$ has generators of type (1). Then the normal closure $N$ of such an $x_{r}(t)$ is $G_{I}^{1}$ where $I=J L_{R}, J=\langle t\rangle$, the principal ideal generated by $t$ in $R$.

Proof. Note that there are no type (3) generators by hypothesis. If we use $\omega_{r}(1)=x_{r}(1) x_{-r}(-1) x_{r}(1)$ corresponding to

$$
w \in W^{1}=\{w \in W \mid w(\bar{s})=\bar{w}(s)\},
$$

where $W$ is the Weyl group, then we can obtain any $x_{s}( \pm t)$ for $s=\bar{s}$ as a member of $N$, in view of Corollary 2.8 of [6]. In addition,

$$
\begin{aligned}
\left(x_{r}(t), x_{s}(1) x_{s}^{-}(1)\right) & =\left(x_{r}(t), x_{s}(1)\right) x_{s}(1)\left(x_{r}(t), x_{s}(1)\right) x_{s}(1)^{-1} \\
& =x_{r+s}( \pm t) x_{s}(1) x_{r+s}( \pm t) x_{s}(1)^{-1} \\
& =x_{s}(1) x_{r+s}( \pm t) x_{r+s}( \pm t) x_{s}(1)^{-1}
\end{aligned}
$$

and hence $x_{r+s}( \pm t) x_{r+\bar{s}}( \pm t)$ is in $N$. Observe that the plus or minus sign is the same in each factor since it is determined by $N_{r, s}=N_{r, \bar{s}}$ since $(r+s)^{-}=r+\bar{s}$. Since $r+s \neq(r+s)^{-}=r+\bar{s}$, we have one type (5) generator in $N$. But then again using elements corresponding to members of $W^{1}$, we get all such type (5) elements in $N$. Since all the generators of $G_{I}^{1}$ as a normal subgroup are in $N$, we have $G_{I}^{1} \subseteq N$, so $G_{I}^{1}=N$, as desired.

\section{REFERENCES}

1. J. Hurley, Ideals in Chevalley algebras, Trans. Amer. Math. Soc. 137 (1969), 245-258. MR 38 \#5870.

2. - Some normal subgroups of elementary subgroups of Chevalley groups over rings, Amer. J. Math. 93 (1971), 1059-1069.

3. - Normality and terminality in the elementary subgroups of Steinberg groups over rings, Proc. Amer. Math. Soc. 34 (1972), 30-34. MR 45 \#8750.

4. W. Klingenberg, Lineare Gruppen über lokalen Ringen, Amer. J. Math. 83 (1961), 137-153. MR 23 \#A1724.

5. W. Scott, Group theory, Prentice-Hall, Englewood Cliffs, N.J., 1964. MR 29 \#4785.

6. R. Steinberg, Variations on a theme on Chevalley, Pacific J. Math. 9(1959), 875-891. MR 22 \#79.

7. - Lectures on Chevalley groups, Mathematics Dept., Yale University, New Haven, Conn., 1967/68.

Department of Mathematics, University of Connecticut, Storrs, Connecticut 06268 